\title{
Molecular Docking Studies on Synthetic Therapeutic Agents for COVID-19 ${ }^{+}$
}

\author{
Lucia Pintilie ${ }^{1, *}$, Constantin Tanase ${ }^{1}$ and Ranjan Kumar Mohapatra ${ }^{2}$ \\ 1 National Institute for Chemical-Pharmaceutical Research and Development (ICCF)-Bucharest, \\ 112 Vitan Av., 031299 Bucharest, Romania; cvtanase@gmail.com \\ 2 Government College of Engineering, Keonjhar At-Jamunalia, Po-Old Town Keonjhar, Odisha 758002, India; \\ ranjan_mohapatra@yahoo.com \\ * Correspondence: lucia.pintilie@gmail.com \\ + Presented at the 24th International Electronic Conference on Synthetic Organic Chemistry, \\ 15 November-15 December 2020; Available online: https://ecsoc-24.sciforum.net/.
}

Citation: Pintilie, L.; Tanase, C.; Mohapatra, R.K. Molecular Docking Studies on Synthetic Therapeutic Agents for COVID-19. Chem. Proc. 2021, 3, 46. https://doi.org/10.3390/ ecsoc-24-08352

Academic Editors: Julio A. Seijas and M. Pilar Vázquez-Tato

Published: 14 November 2020

Publisher's Note: MDPI stays neutral with regard to jurisdictional claims in published maps and institutional affiliations.

Copyright: (๑) 2020 by the authors. Licensee MDPI, Basel, Switzerland. This article is an open access article distributed under the terms and conditions of the Creative Commons Attribution (CC BY) license (http://creativecommons.org/licenses/by/4.0/).

\begin{abstract}
Coronavirus disease (COVID-19) is an infectious disease caused by coronavirus 2 (SARSCoV-2) which was detected for the first time in Wuhan China in December 2019. The rapid spread of this highly contagious and pathogenic virus led to the declaration of the pandemic by the World Health Organization (WHO) on March 11, 2020. In these conditions, the discovery of new antiviral agents is extremely important. For the development of the anti-SARS-CoV-2 drugs, the fastest way is to find potential molecules from the marketed drugs by molecular docking studies.
\end{abstract}

Keywords: COVID-19; SARS-CoV-2; molecular docking; anti-viral agents; anti-inflammatory agents

\section{Introduction}

Coronavirus disease (COVID-19) is an infectious disease caused by coronavirus 2 (SARS-CoV-2) which was detected for the first time in Wuhan China in December 2019 [1]. The rapid spread of this highly contagious and pathogenic virus led to the declaration of the pandemic by the World Health Organization (WHO) on 11 March 2020.

The scientific community around the world is concerned with finding an effective treatment for the new coronavirus. Short-term efforts are focused on developing vaccines or inhibitors that act as protection against infection with the new coronavirus [2].

To develop new drugs with antiviral activity, the concern of many research groups is focused on the repositioning of already approved drugs [3-6]. The fastest way is to find potential drugs by molecular docking studies [7-13].

\section{Molecular Docking Studies}

Molecular docking studies have been performed to identify and visualize the most likely interaction of the ligand with the protein receptor [14]. The docking score and hydrogen bonds formed with the amino acids from of the group interaction atoms are used to predict the binding modes, the binding affinity, and the orientation of the docked ligands in the active site of the protein/enzyme receptor. The docking study have been carried out with synthetic anti-viral agents (13) [15-18] and anti-inflammatory agents (2) [5] (Figure 1), prepared using Spartan 14 Software [15].

In this study are five investigated SARS-CoV-2 targets: main protease (PD ID: 6W63, PD ID: 6WNP), spike glycoprotein (closed state) (PD ID: 6VXX), chimeric receptor-binding domain complexed with its receptor human ACE2 (PD ID: 6VW1), RNA-dependent RNA polymerase (PD ID: 6M71) and 3CL protease (3CL pro) (PD ID: 6M2N). 


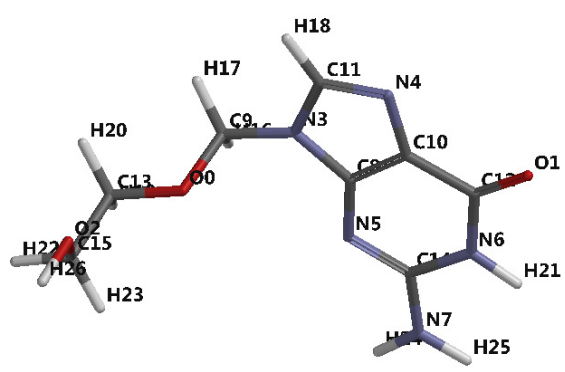

Acyclovir

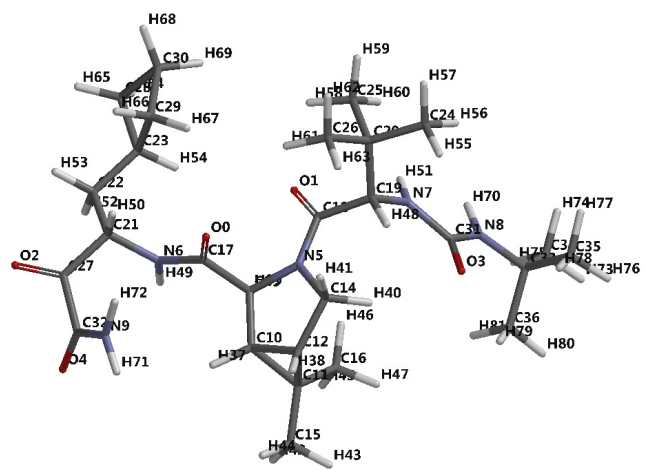

Boceprevir

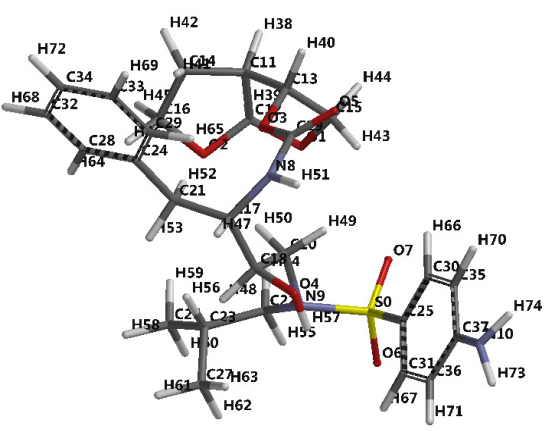

Darunavir

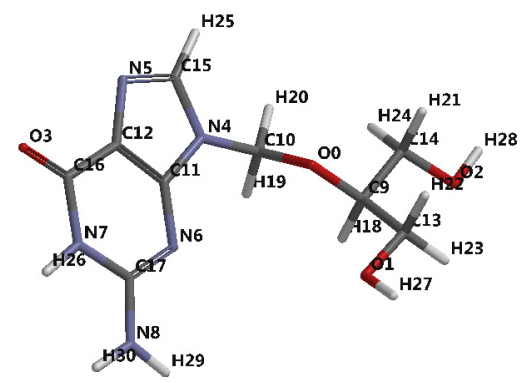

Ganciclovir

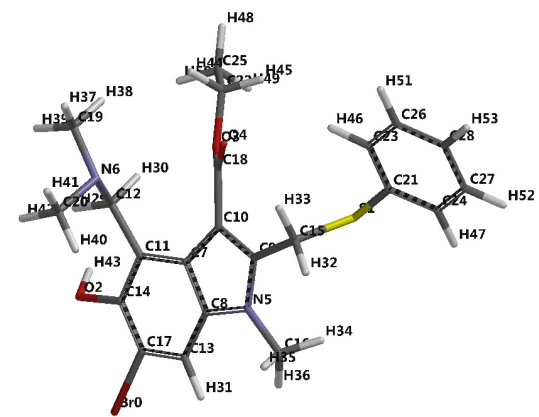

Arbidol

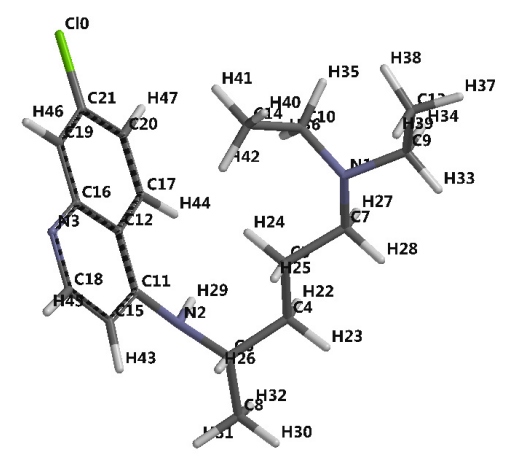

Chloroquine

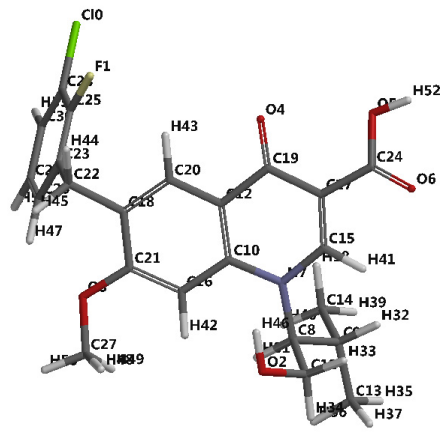

Elvitegravir

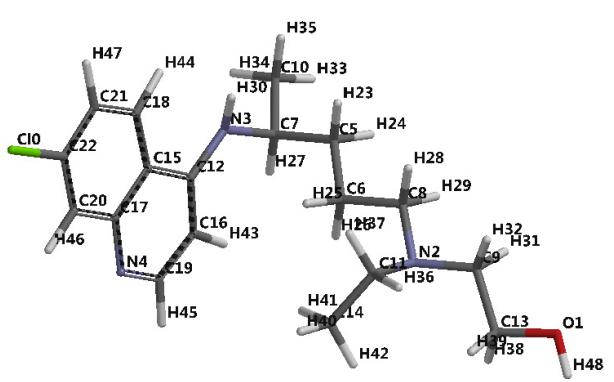

Hydroxychloroquine 


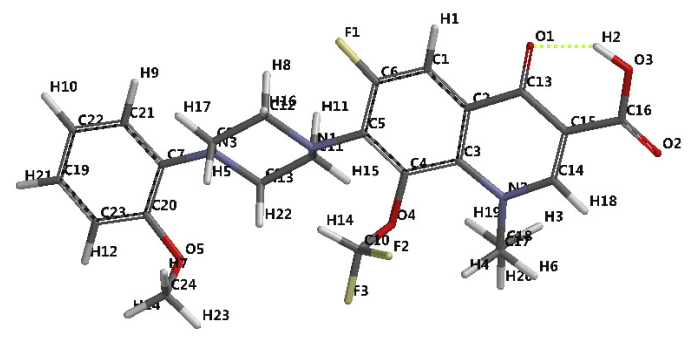

$\mathrm{K}-12$

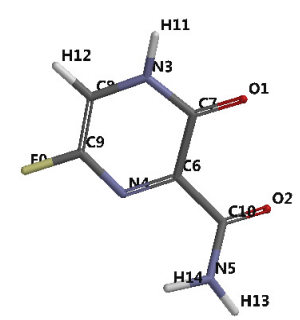

Favipiravir

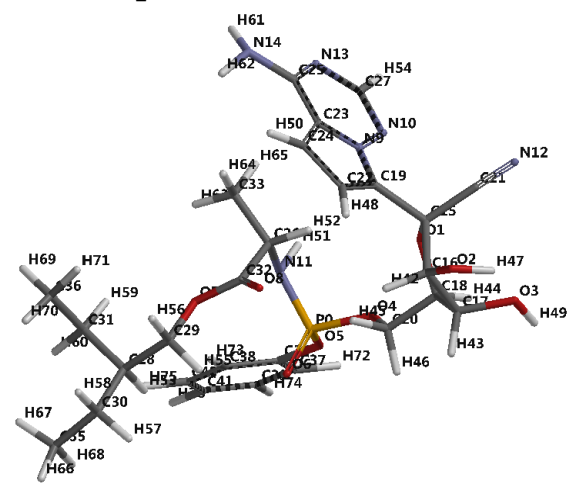

Remdesivir

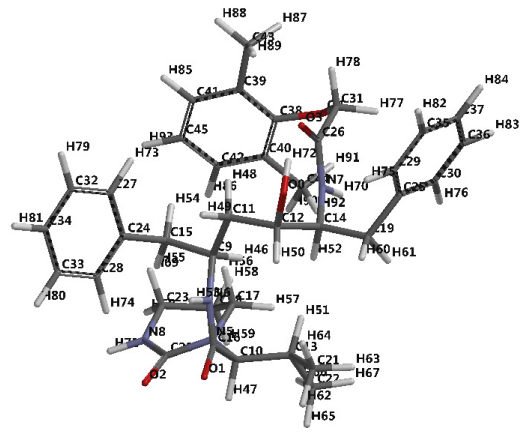

Lopinavir

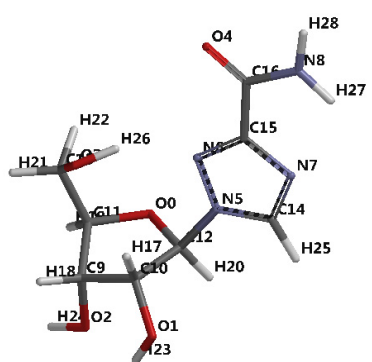

Ribavirin

\section{Oseltamivir}

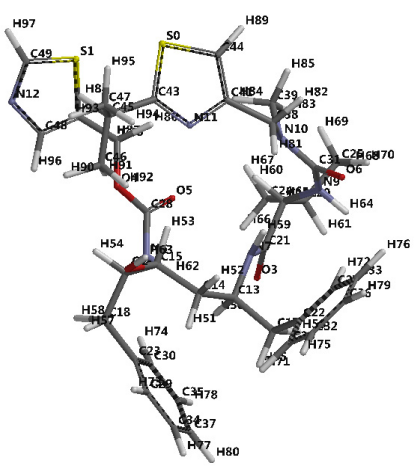

Ritonavir

Figure 1. Tube representation of the optimized molecular structure of ligands. The numbering of the atoms was done according to the software.

\subsection{SARS-CoV-2 Main Protease}

\subsubsection{SARS-CoV-2 Main Protease Receptor PD ID: 6W63}

Docking studies were realized to obtain accurate predictions of the optimized conformations for both the ligands and protein target to form a stable complex. All compounds were docked on the crystal structure of SARS-CoV-2 main protease (PDB ID: $6 \mathrm{~W} 63)$. The docking pose of the co-crystallized X77 interacting with amino acid residues of the active site and the hydrogen bonds created with GLU 166 (2.721 $\AA$ ) and GLY 143 $(3.202 \AA)$ are shown in Figure 2. The co-crystallized X77 (N-(4-tert-butylphenyl)-N[(1R)-2-(cyclohexylamino)-2-oxo-1-(pyridin-3-yl)ethyl]-1H-imidazole -4-carboxamide) was taken as a reference ligand to compare the docking results of the studied compounds. The docking studies revealed that the docking scores of ten compounds are better than that of the co-crystallized X77 (docking score: -56.65; RMSD: $0.90 \AA$ ) (root mean square deviations) (Table 1, Figure 3): Ritonavir (docking score: -93.42; RMSD: $2.98 \AA$ ), Lopinavir (docking score: -83.15; RMSD: $2.15 \AA$ ), Remdesivir (docking score: -76.90; RMSD: 1.89 $\mathrm{A}$ ), Darunavir (docking score: -70.33; RMSD: $2.14 \AA$ ), Elvitegravir (docking score: -67.97; RMSD: $0.23 \AA$ ), Arbidol (docking score: -63.32; RMSD: $0.23 \AA$ ), K-12 (docking score: 
-63.05; RMSD: $0.32 \AA$ ), Hydroxychloroquine (docking score: -62.66; RMSD: $0.86 \AA$ ), Chloroquine (docking score: -59.17; RMSD: $0.85 \AA$ ), and Boceprevir (docking score: -58.78; RMSD: $4.05 \AA$ ). In order to have a prediction as close to reality as possible, the RMSD value must be less than $2 \AA$ [19]; for the compounds Ritonavir, Lopinavir, Darunavir and Boceprevir, it can be considered that the prediction is not very accurate. The compound Remedesivir (docking score: -76.90; RMSD: 1.89 $\AA$ ) shows the occurrence of five hydrogen bonds with: GLU 166 (3.070 ̊), HIS 141 (3.205 $)$ ), LEU 141 (2.651 $\AA$ ), SER 144 (2.768 ̊̊) and HIS 163 (3.039 ̊) (Figure 4a).

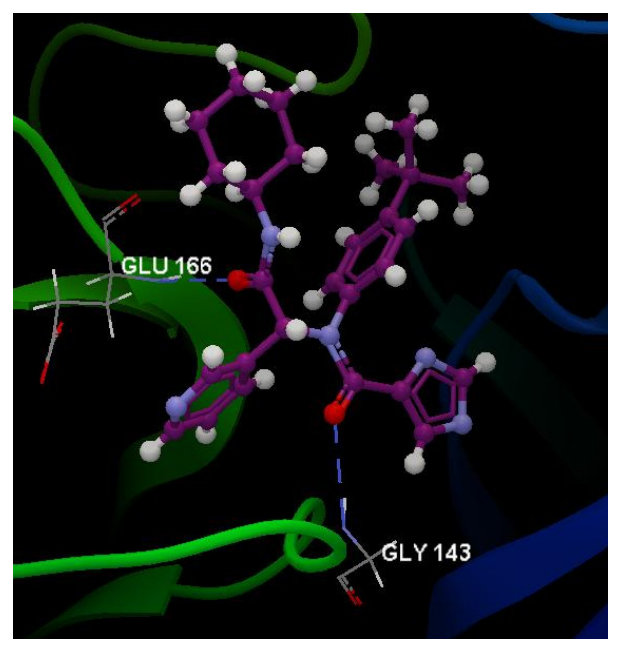

Figure 2. Hydrogen bonds between co-crystallized X77 and GLU 166 and GLY 143 amino acids residues of the binding site of $6 \mathrm{~W} 63$.

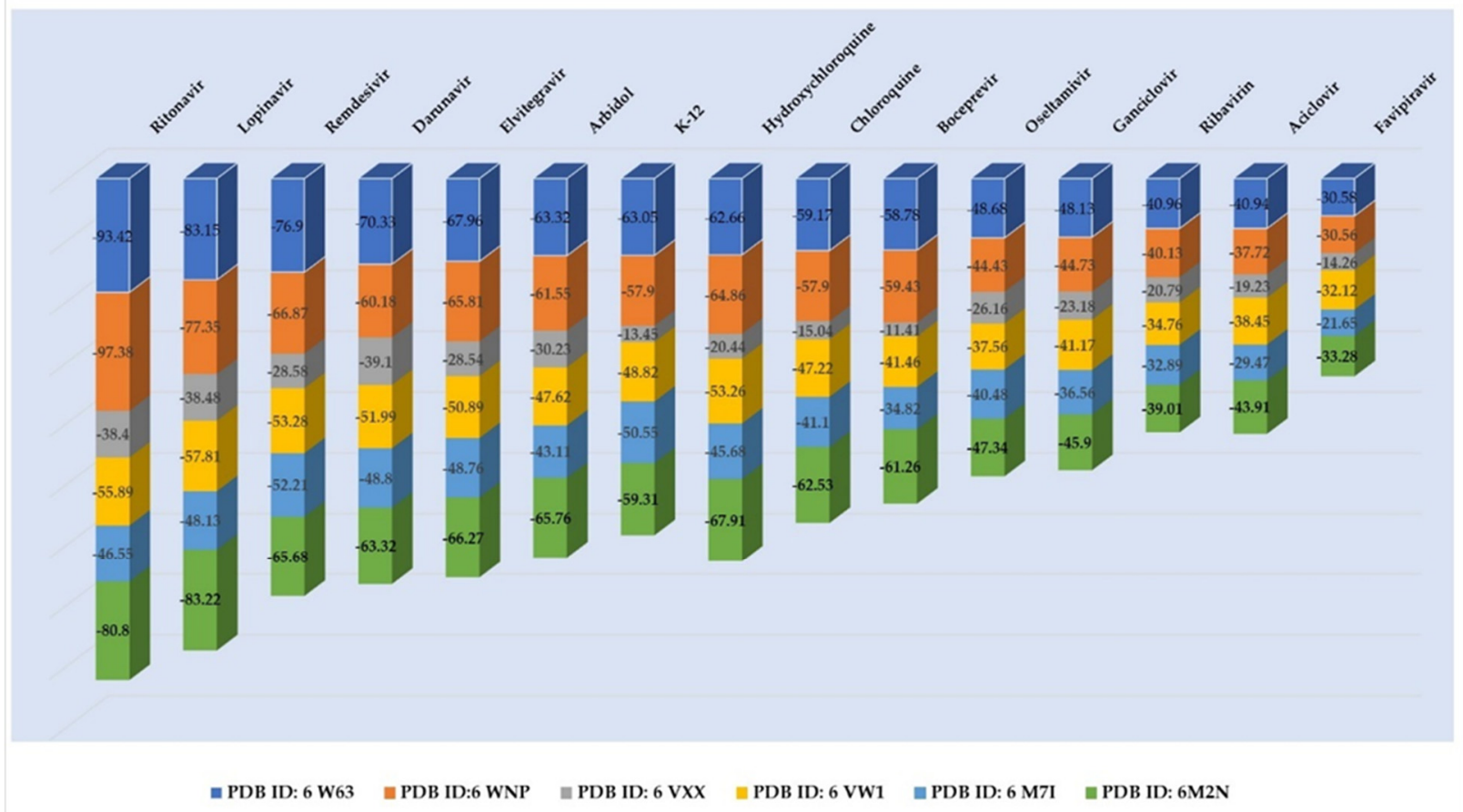

Figure 3. Docking score of the studied molecules. 


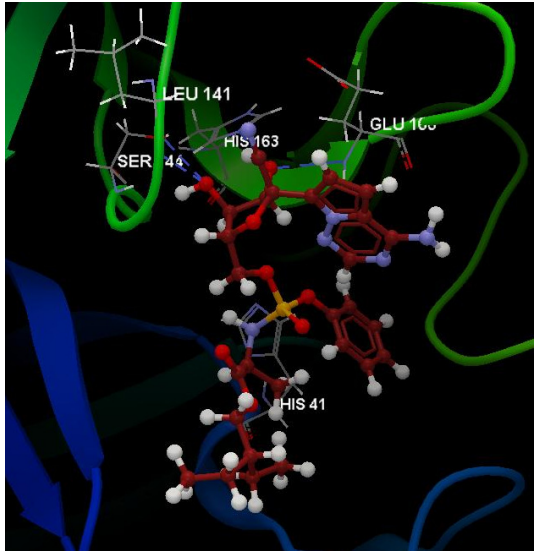

(a)

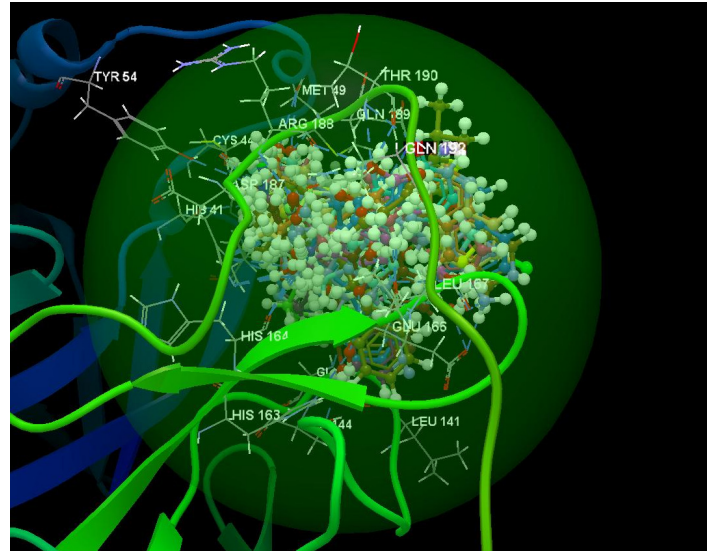

(b)

Figure 4. (a) Hydrogen bonds between the Remdesivir ligand interacting with the GLU 166, HIS 141, LEU 141, SER 144 and HIS 163 amino acid residues of the binding site of 6W63. (b)Docking pose of the co-crystallized X77 and all ligands in the binding site of 6W63.

After analyzing the docking study results, it was observed that all the studied ligands were placed in the same binding site (green sphere) of $6 \mathrm{~W} 63$ as the co-crystallized X77, and it wasobserved they have the same orientation as the co-crystallized ligand (Figure $4 \mathrm{~b}$ ).

\subsubsection{SARS-CoV-2 Main Protease Receptor PD ID: 6WNP}

Docking studies were realized to obtain accurate predictions of the optimized conformations for both the ligands and protein target to form a stable complex. All the compounds were docked on the crystal structure of the SARS-CoV-2 main protease (PDB ID: 6WNP). The docking pose of the co-crystallized USG A 101 interacting with amino acid residues of the active site and the hydrogen bonds created with CYS 145 (2.900 $\AA$ ),

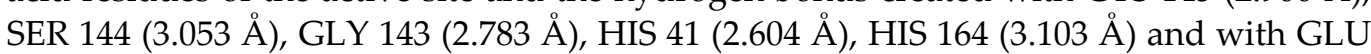
166 (3.118 $\AA$, $2.908 \AA, 3.229 \AA$ ) are shown in Figure 5a. The co-crystallized USG A 101 (Boceprevir -bound form) was taken as a reference ligand to compare the docking results of the studied compounds. The docking studies revealed that the docking scores of five compounds are better than that of the co-crystallized USG A 101 (docking score: -63.95; RMSD: $0.80 \AA$ ) (Table 1, Figure 3): Ritonavir (docking score: -97.38; RMSD: $3.54 \AA$ ), Lopinavir (docking score: -73.55; RMSD: $3.48 \AA$ A), Remdesivir (docking score: -66.87; RMSD: $1.52 \AA$ ), Elvitegravir (docking score: -65.81; RMSD: $0.23 \AA$ ), and Hydroxychloroquine (docking score: -64.86 ; RMSD: $1.25 \AA$ ). In order to have a prediction as close to reality as possible, the RMSD value must be less than $2 \AA$ [19]; for compounds Ritonavir and Lopinavir, it can be considered that the prediction is not very accurate. The compound Renedesivir (docking score:-66.87; RMSD: $1.52 \AA$ ) shows the occurrence of four hydrogen bonds: two with GLU 166 (3.080 ̊̊ and $3.156 \AA$ ) and two with GLN 189 (2.936 ̊ and 2.925) (Figure 5b). 
Table 1. Docking score of ligands.

\begin{tabular}{|c|c|c|c|c|c|c|c|c|c|c|c|c|}
\hline \multirow{2}{*}{ Ligand } & \multicolumn{2}{|c|}{ PDB ID: 6W63 } & \multicolumn{2}{|c|}{ PDB ID: 6WNP } & \multicolumn{2}{|c|}{ PDB ID: 6VXX } & \multicolumn{2}{|c|}{ PDB ID: 6VW1 } & \multicolumn{2}{|c|}{ PDB ID: 6M71 } & \multicolumn{2}{|c|}{ PDB ID:6M2N } \\
\hline & Score & RMSD [Å] & Score & RMSD [Å] & Score & RMSD [Å] & Score & RMSD[Å] & Score & RMSD[Å] & Score & RMSD[Å] \\
\hline Co-crystallized & -56.65 & 0.90 & -63.95 & 0.80 & -18.42 & 0.51 & -32.77 & 0.20 & - & - & -53.49 & 0.37 \\
\hline Ritonavir & -93.42 & 2.98 & -97.38 & 3.54 & -38.40 & 5.17 & -55.89 & 3.16 & -46.55 & 2.49 & -80.80 & 3.24 \\
\hline Lopinavir & -83.15 & 2.15 & -77.35 & 3.48 & -38.48 & 4.69 & -57.81 & 2.67 & -48.13 & 2.35 & -83.22 & 0.98 \\
\hline Remdesivir & -76.90 & 1.89 & -66.87 & 1.52 & -28.58 & 3.78 & -53.28 & 2.95 & -52.21 & 2.28 & 65.68 & 1.33 \\
\hline Darunavir & -70.33 & 2.14 & -60.18 & 1.84 & -39.10 & 4.06 & -51.99 & 1.94 & -48.80 & 1.34 & -63.32 & 1.94 \\
\hline Elvitegravir & -67.96 & 0.23 & -65.81 & 0.58 & -28.54 & 2.10 & -50.89 & 0.40 & -48.76 & 0.06 & -66.27 & 0.07 \\
\hline Arbidol & -63.32 & 0.23 & -61.55 & 0.48 & -30.23 & 1.38 & -47.62 & 0.87 & -43.11 & 0.49 & -65.76 & 0.04 \\
\hline $\mathrm{K}-12$ & -63.05 & 0.32 & -57.90 & 0.61 & -13.45 & 1.57 & -48.82 & 0.63 & -50.55 & 0.40 & -59.31 & 0.52 \\
\hline Hydroxychloroquine & -62.66 & 0.86 & -64.86 & 1.25 & -20.44 & 3.08 & -53.26 & 0.71 & -45.68 & 1.06 & -67.91 & 1.07 \\
\hline Chloroquine & -59.17 & 0.85 & -57.90 & 1.07 & -15.04 & 3.71 & -47.22 & 1.17 & -41.10 & 0.96 & -62.53 & 1.85 \\
\hline Boceprevir & -58.78 & 4.05 & -59.43 & 0.80 & -11.41 & 4.15 & -41.46 & 1.41 & -34.82 & 1.07 & -61.26 & 1.80 \\
\hline Oseltamivir & -48.68 & 0.51 & -44.43 & 0.45 & -26.16 & 3.82 & -37.56 & 0.63 & -40.48 & 1.25 & -47.34 & 0.48 \\
\hline Ganciclovir & -48.13 & 0.81 & -44.73 & 0.55 & -23.18 & 0.80 & -41.17 & 0.62 & -36.56 & 0.48 & -45.90 & 0.25 \\
\hline Ribavirin & -40.96 & 0.03 & -40.13 & 0.75 & -20.79 & 0.19 & -34.76 & 0.08 & -32.89 & 0.02 & -39.01 & 0.03 \\
\hline Acyclovir & -40.94 & 0.77 & -37.72 & 0.72 & -19.23 & 1.15 & -38.45 & 0.22 & -29.47 & 0.52 & -43.91 & 0.04 \\
\hline Favipiravir & -30.58 & 0.03 & -30.56 & 0.01 & -14.26 & 0.13 & -32.12 & 0.01 & -21.65 & 0.04 & -33.28 & 0.09 \\
\hline
\end{tabular}




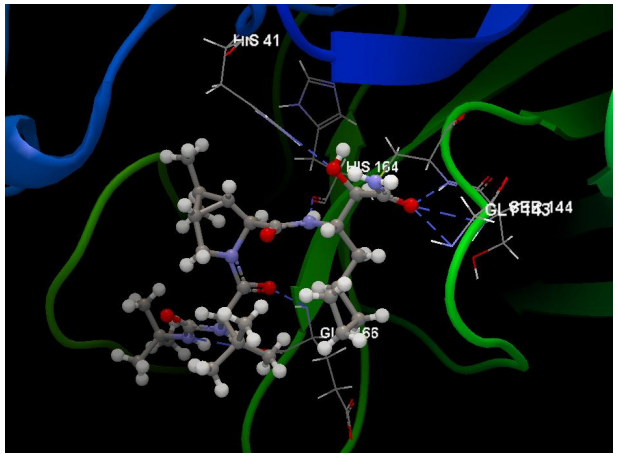

(a)

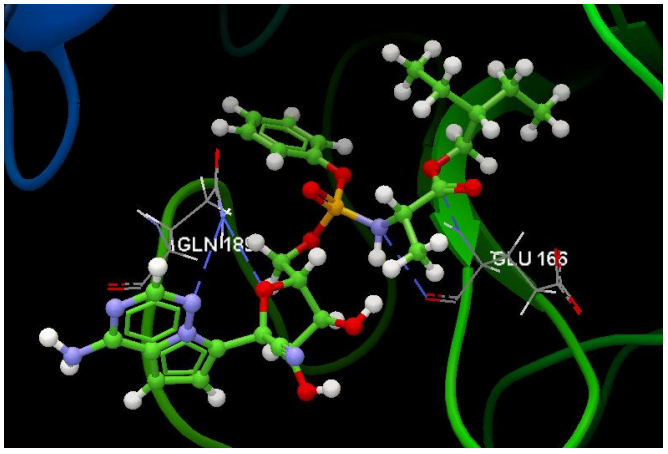

(b)

Figure 5. (a). Hydrogen bonds between co-crystallized USG A 101 and GLU 166, CYS 145, SER 144, GLY 143, HIS 141 and HIS 164 amino acids residues of the binding site of 6WNP. (b) Hydrogen bonds between the Remdesivir interacting with the GLU 166 and GLN 189 amino acid residues of the binding site of $6 \mathrm{WNP}$.

The docking study results reveals that all of the studied ligands were placed in the same binding site (green sphere) of 6WNP as the co-crystallized USG A 101, and it was observed that they have the same orientation as the co-crystallized ligand (Figure 6); it was also observed that the correlation of the results obtained for two X-ray structures of SARS-CoV-2 main protease (6W63 and 6WNP) were downloaded from PDB bank (Protein data bank).

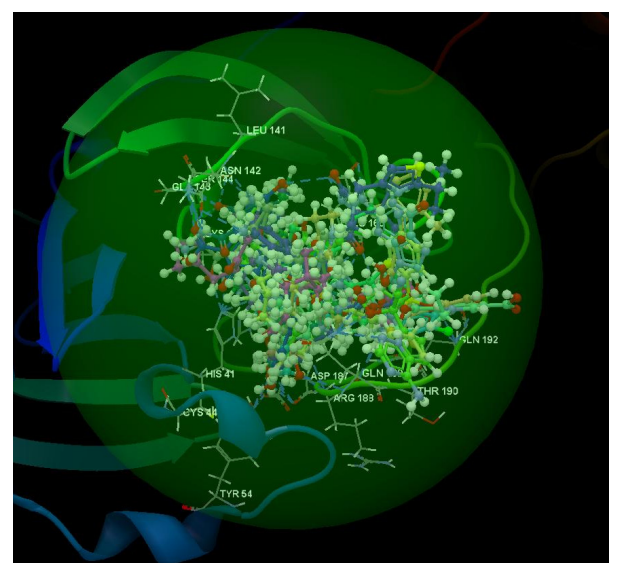

Figure 6. Docking pose of the co-crystallized USG A 101 and all ligands in the binding site of 6WNP.

\subsection{SARS-CoV-2 Spike Glycoprotein}

All the compounds were docked on the crystal structure of SARS-CoV-2 spike glycoprotein (closed state) (PD ID: 6VXX).The docking pose of the co-crystallized NAG 1302 interacting with the amino acid residues of the active site and the hydrogen bonds created with ASN 122 (2.393 $\AA, 2.441 \AA$ and $2.449 \AA$ ) are shown in Figure 7a. The co-crystallized NAG 1302 was taken as a reference ligand to compare the docking results of the studied compounds. The docking studies revealed that the docking scores of four compounds are smaller than that of the co-crystallized NAG 1302 (docking score: -18.42; RMSD: $0.51 \AA$ ) (Table 1, Figure 3): Chloroquine (docking score: -15.04; RMSD: 3.71 Å), Favipavir (docking score: -14.26; RMSD: $0.13 \AA$ ), K-12 (docking score: -13.45; RMSD: $1.57 \AA$ ) and Boceprevir (docking score: -11.41 ; RMSD: $4.15 \AA$ ). Darunavir has the best docking score (docking score: -39.10; RMSD: $4.06 \AA$ ). Darunavir is the only compound who shows the occurrence of the hydrogen bonds with the same ASN 122 amino acid (3.092 $\AA, 2.812 \AA$ and $2.950 \AA$ ) like co-crystallized NAG 1302 (Figure 7b). Because the RMSD value is greater than $2 \AA$ [19], the prediction for Darunavir is not very accurate in this case. 


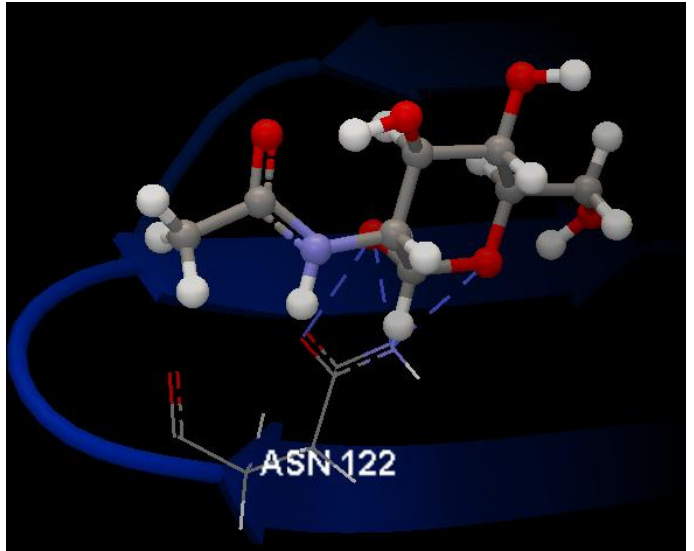

(a)

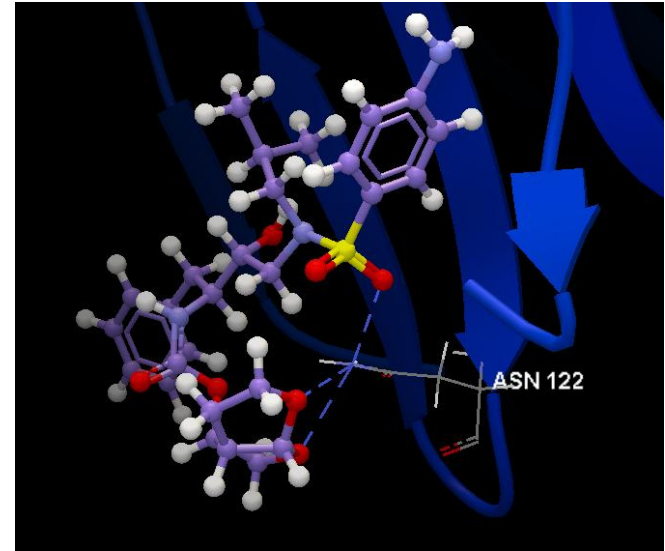

(b)

Figure 7. (a) Hydrogen bonds between co-crystallized NAG 1302 and ASN 122 amino acid residue of the binding site of 6VXX. (b) Hydrogen bonds between the Darunavir interacting with the ASN 122 amino acid residue of the binding site of $6 \mathrm{VXX}$.

The docking study results reveal that all of the studied ligands were placed in the same binding site (green sphere) of 6VXX as the co-crystallized NAG 1302 but only Darunavir had the same orientation as the co-crystallized ligand (Figure 8).

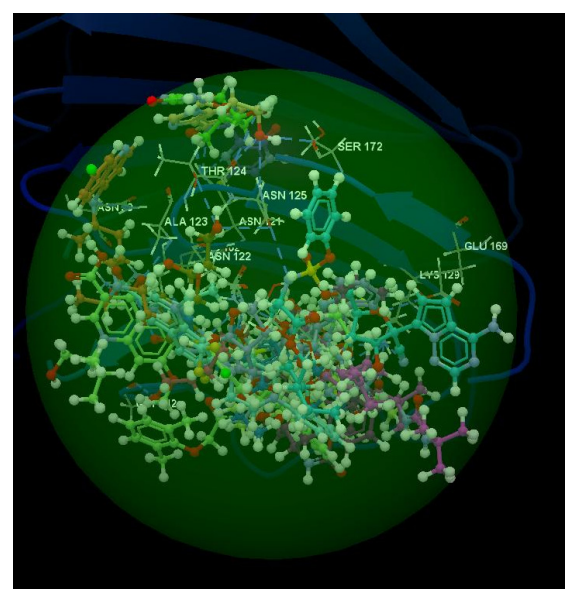

Figure 8. Docking pose of the co-crystallized NAG 1302 and all ligands in the binding site of 6VXX.

\subsection{SARS-CoV-2 Chimeric Receptor-Binding Domain Complexed with Its Receptor Human ACE2}

All the compounds are docked on the crystal structure of SARS-CoV-2 chimeric receptor-binding domain complexed with its receptor human ACE2 (PD ID: 6VW1). The docking pose of the co-crystallized NAG 714 interacting with amino acid residues of the active site and the hydrogen bonds created with ASN 103 (2.434 $\AA, 2.310 \AA$ and $2.562 \AA$ ) and GLN 81 (2.914 $\AA$ and $2.602 \AA$ ) are shown in Figure 9a. The co-crystallized NAG 714 was taken as a reference ligand to compare the docking results of the studied compounds. The docking studies revealed that only Favipavir (docking score: -32.12; RMSD: $0.01 \AA$ ) has a docking score smaller than that of the co-crystallized NAG 714 (docking score: -32.77; RMSD: $0.20 \AA$ ) (Table 1, Figure 3). Remdesivir compound (docking score: -51.99 ; RMSD: $2.95 \AA$ ) shows the occurrence of hydrogen bonds with the same amino acids ASN 103 (3.007 $\AA$, $3.112 \AA$ and $3.057 \AA$ ) and GLN 81 (3.103 $\AA$ ) like co-crystallized NAG 714 and

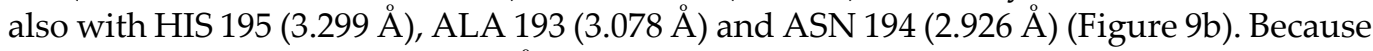
the RMSD value is greater than $2 \AA$ [19], the prediction for Remdesivir is not very accurate in this case. 


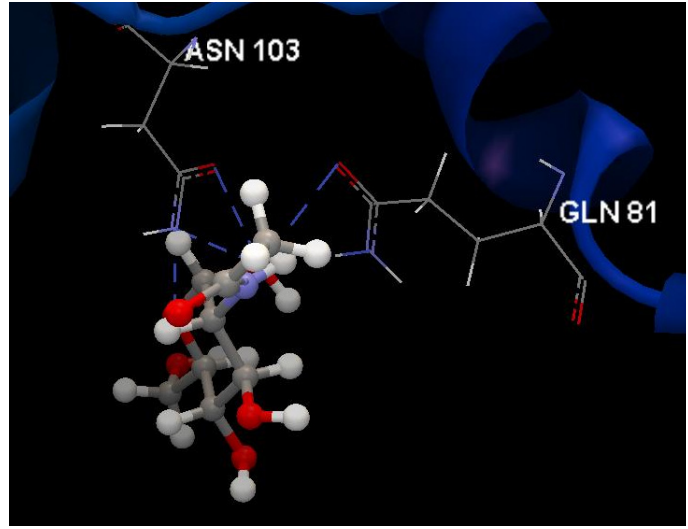

(a)

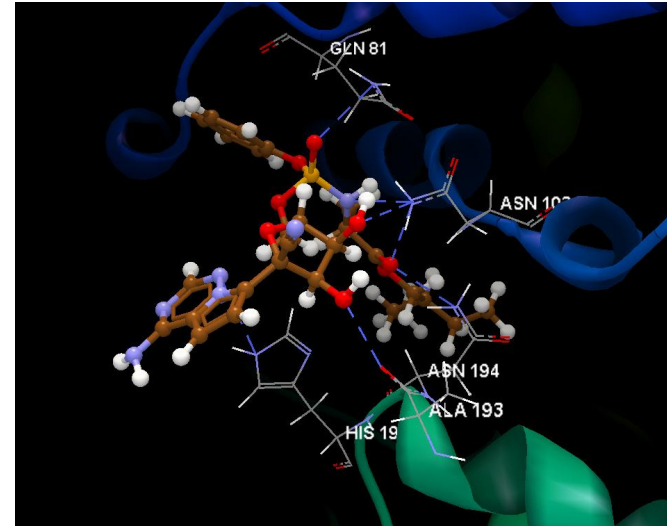

(b)

Figure 9. (a). Hydrogen bonds between co-crystallized NAG 714 and ASN 103 and GLN 81 amino acid residues of the binding site of 6VW1. (b) Hydrogen bonds between the Remdesivir interacting with the ASN 103, GLN 81, HIS 195, ALA 193 and ASN 194 amino acids residues of the binding site of 6VW1.

The docking study results reveal that all of the studied ligands were placed in the same binding site (green sphere) of 6VW1 as the co-crystallized NAG 714, and it was observed they have the same orientation as the co-crystallized ligand (Figure 10).

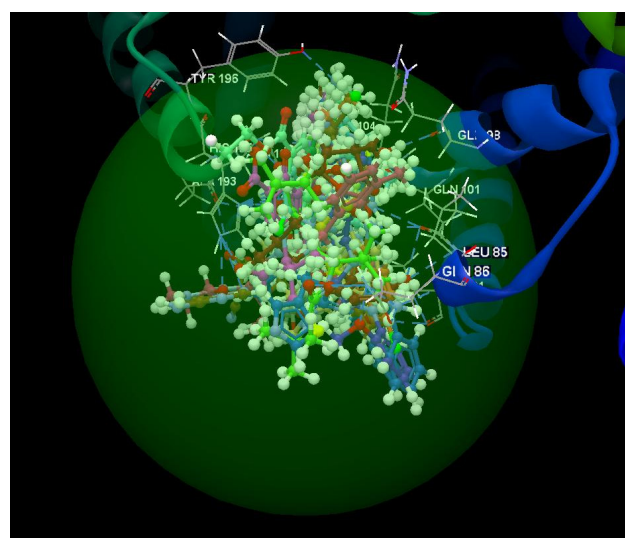

Figure 10. Docking pose of the co-crystallized NAG 714 and all ligands in the binding site of 6VW1.

\subsection{SARS-CoV-2 RNA-Dependent RNA Polymerase}

All compounds were docked on the crystal structure of SARS-CoV-2 RNA-dependent RNA polymerase (PD ID: 6M71). The docking studies revealed that Remdesivir has the best docking score: -52.51 (RMSD: 2.28) (Table 1, Figure 4) and shows the occurrence of six hydrogen bonds with the ASP 118 (2.792 $\AA$ ), ASP 760 (2.643 $)$ ), ASN $691(2.705 \AA)$, ASP $623(3.320 \AA)$, CYS 522 (3.348 $)$ ) and LYS 621 (2950 A) (Figure 11a). Because the RMSD value is greater than $2 \AA$ [19], the prediction for Remdesivir is not very accurate in this case. All studied ligands were placed in the same binding site (green sphere) of 6M71 (Figure 11b). 


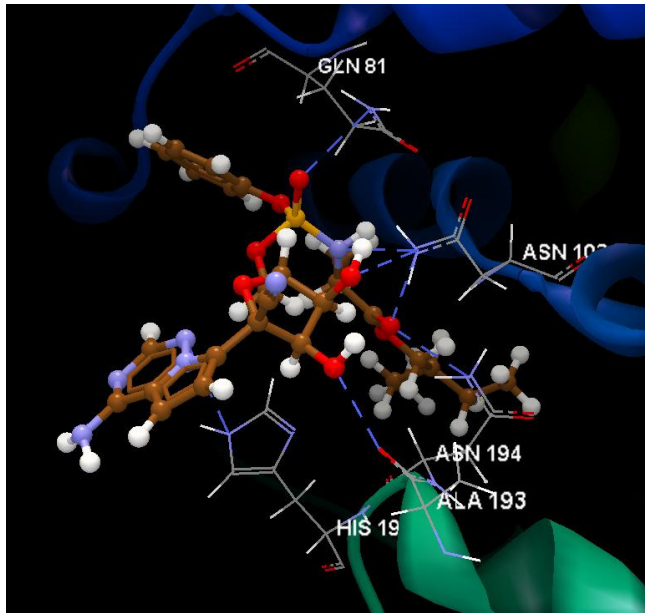

(a)

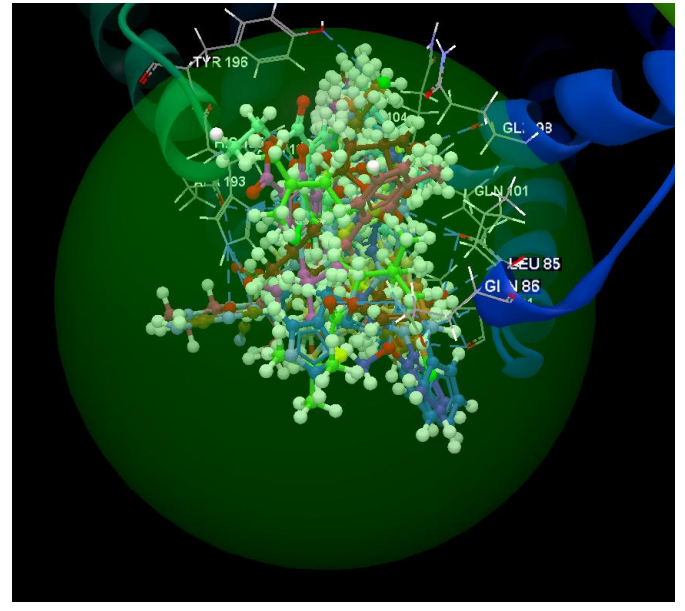

(b)

Figure 11. (a) Hydrogen bonds between the Remdesivir interacting with the ASP 118, ASP 760, ASN 691, ASP 623, CYS 522 and LYS 621. Amino acids residues of the binding site of 6M71. (b) Docking pose of all ligands in the binding site of $6 \mathrm{M} 71$.

\subsection{SARS-CoV-2 3CL Protease (3CL pro)}

Docking studies were realized to obtain accurate predictions of the optimized conformations for both the ligands and protein target to form a stable complex. All compounds were docked on the crystal structure of SARS-CoV-2 3CL protease (3CL pro) (PD ID: 6M2N). The docking pose of the co-crystallized 3WL A interacting with the amino acid residues of the active site and the hydrogen bonds created with GLU 166 (3.016 $\AA$ ), GLY 143 (3.104 $\AA$ and $2.969 \AA$ ) and ASN 142 (3.341 $\AA$ ) are shown in Figure 12a. The cocrystallized $3 \mathrm{WL}$ A was taken as a reference ligand to compare the docking results of the studied compounds. All compounds except Oseltamivir, Ganciclovir, Aciclovir, Ribavirin and Favipavir have a docking score greater than co-crystallized 3WL A (docking score: -53.49; RMSD: $0.37 \AA$ ) (Table 1, Figure 3). Lopinavir has the best docking score: -83.22 (RMSD: $0.98 \AA$ ) and shows the occurrence of hydrogen bonds with the same amino acids GLU 166 (3.013 ̊), GLY $143(2.637 \AA)$ and ASN 142 (2.944 ̊̊) like co-crystallized 3WL A (Figure 12b).

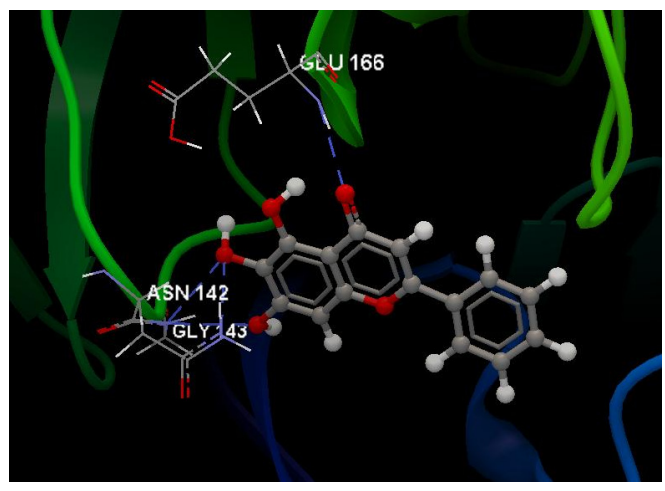

(a)

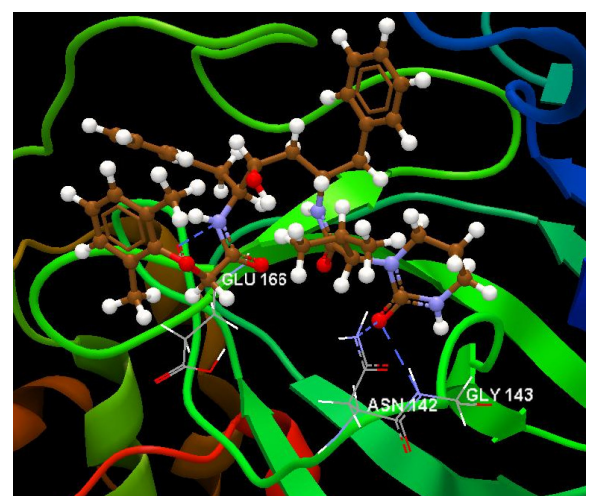

(b)

Figure 12. (a) Hydrogen bonds between co-crystallized 3WL A and GLU 166, GLY 143 and ASN 142 amino acids residues of binding. Site of 6M2N. (b) Hydrogen bonds between the Lopinavir ligand interacting with the GLU 166, GLY 143 and ASN 142 amino acid residues of the binding site of $6 \mathrm{M} 2 \mathrm{~N}$.

After analyzing the docking study results, it was observed that all of the studied ligands were placed in the same binding site (green sphere) of $6 \mathrm{M} 2 \mathrm{~N}$ as the co-crystallized 
3WL $A$ and it was observed they have the same orientation as the co-crystallized ligand (Figure 13).

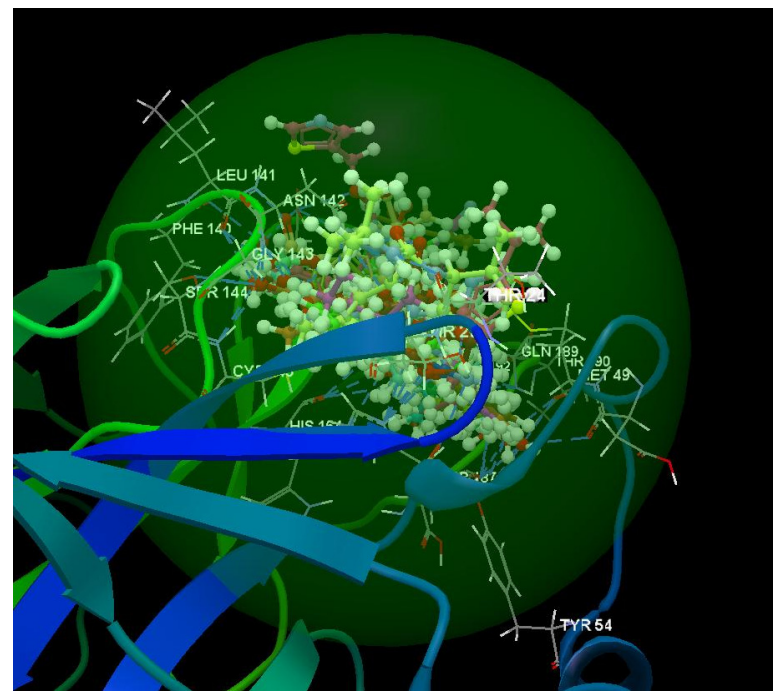

Figure 13. Docking pose of the co-crystallized 3WL $\mathrm{A}$ and all ligands in the binding site of $6 \mathrm{M} 2 \mathrm{~N}$.

\section{Conclusions}

The docking study was carried out with synthetic anti-viral agents (13) and anti-inflammatory agents (2) as ligands against the SARS-CoV-2 main protease (PD ID: 6W63, PD ID: 6WNP), SARS-CoV-2 spike glycoprotein (closed state) (PD ID: 6VXX), SARS-CoV2 chimeric receptor-binding domain complexed with its receptor human ACE2 (PD ID: 6VW1), SARS-CoV-2 RNA-dependent RNA polymerase (PD ID: 6M71), and SARS-CoV-2 $3 \mathrm{CL}$ protease (3CL pro) (PD ID: 6M2N).

The study indicates the possibility of using approved drugs in the treatment of coronavirus disease (COVID-19).

The best results were obtained for antiretrovirals:

- protease inhibitors: Lopinavir, Ritonavir, Darunavir

- integrase inhibitors: Elvitegravir and Remdesivir, originally developed for the treatment of Marburg virus, Ebola virus and Cueva virus infections.

Author Contributions: Conceptualization, L.P., C.T. and R.K.M.; software, L.P.; writing and editing, L.P.; visualization, C.T. and R.K.M.; all authors have read and agreed to the published version of the manuscript.

Funding: This paper has been financed through the NUCLEU Program, which is implemented with the support of ANCSI project no. PN 19-41 0102.

Institutional Review Board Statement:-Not applicable.

Informed Consent Statement:-Not applicable.

Data Availability Statement:-Not applicable.

Conflicts of Interest: The authors declare no conflict of interest.

\section{References}

1. Mohapatra, R.K.; Pintilie, L.; Kandi, V.; Sarangi, A.K.; Das, D.; Sahu, R.; Perekhoda, L. The recent challenges of highly contagious COVID-19, causing respiratory infections: Symptoms, diagnosis, transmission, possible vaccines, animal models, and immunotherapy. Chem. Biol. Drug Des. 2020, doi:10.1111/cbdd.13761.

2. Naqvi, A.A.T.; Fatima, K.; Mohammad, T.; Fatima, U.; Singh, I.K.; Singh, A.; Atif, S.M.; Hariprasad, G.; Hasan, G.M.; Hassan, I. Insights into SARS-CoV-2 genome, structure, evolution, pathogenesis and therapies: Structural genomics approach. Biochim. Biophys. Acta (BBA) Mol. Basis Dis. 2020, 1866, 165878, doi:10.1016/j.bbadis.2020.165878. 
3. Wu, R.; Wang, L.; Kuo, H.-C.D.; Shannar, A.; Peter, R.; Chou, P.J.; Li, S.; Hudlikar, R.; Liu, X.; Liu, Z.; et al. An Update on Current Therapeutic Drugs Treating COVID-19. Curr. Pharmacol. Rep. 2020, 6, 56-70, doi:10.1007/s40495-020-00216-7.

4. Amin, S.A.; Jha, T. Fight against novel coronavirus: A perspective of medicinal chemists. Eur. J. Med. Chem. 2020, 201, 112559, doi:10.1016/j.ejmech.2020.112559.

5. de Almeida, S.M.V.; Santos Soares, J.C.; dos Santos, K.L.; Alves, J.E.F.; Ribeiro, A.G.; Jacob, I.T.T.; da Silva Ferreira, C.J.; dos Santos, J.C.; de Oliveira, J.F.; de Carvalho Junior, L.B.; et al. COVID-19 therapy: What weapons do we bring into battle? Bioorg. Med. Chem. 2020, 28, 115757.

6. Li, G.; De Clercq, E. Therapeutic options for the 2019 novel coronavirus (2019-nCoV). Nat. Rev. Drug Discov. 2020, 19, 149-150, doi:10.1038/d41573-020-00016-0.

7. Pillaiyar, T.; Meenakshisundaram, S.; Manickam, M. Recent discovery and development of inhibitors targeting coronaviruses. Drug Discov. Today 2020, 25, 668-688, doi:10.1016/j.drudis.2020.01.015.

8. ElFiky, A.A. Anti-HCV, nucleotide inhibitors, repurposing against COVID-19. Life Sci. 2020, 248, 117477, doi:10.1016/j.lfs.2020.117477.

9. ElFiky, A.A. Corrigendum to "Ribavirin, Remdesivir, Sofosbuvir, Galidesivir, and Tenofovir against SARSCoV-2 RNA dependent RNA polymerase (RdRp): A molecular docking study\&rdquo; [Life Sci. 253 (2020) 117592]. Life Sci. 2020, 253, 118350, doi:10.1016/j.lfs.2020.118350.

10. Wang, J.; Fast Identification of Possible Drug Treatment of Coronavirus Disease-19 (COVID-19) through Computational Drug Repurposing Study; J. Chem. Inf. Model. 2020, 60, 3277-3286, doi:10.1021/acs.jcim.0c00179.

11. Wu, C.; Liu, Y.; Yang, Y.; Zhang, P.; Zhong, W.; Wang, Y.; Wang, Q.; Xu, Y.; Li, M.; Li, X.; et al. Analysis of therapeutic targets for SARS-CoV-2 and discovery of potential drugs by computational methods. Acta Pharm. Sin. B 2020, 10, 766-788, doi:10.1016/j.apsb.2020.02.008.

12. Yu, R.; Chen, L.; Lan, R.; Shen, R.; Li, P. Computational screening of antagonists against the SARS-CoV-2 (COVID-19) coronavirus by molecular docking. Int. J. Antimicrob. Agents 2020, 56, 106012, doi:10.1016/j.ijantimicag.2020.106012.

13. Cardoso, W.B.; Mendanha, S.A. Molecular dynamics simulation of docking structures of SARS-CoV-2 main protease and HIV protease inhibitors. J. Mol. Struct. 2021, 1225, 129143, doi:10.1016/j.molstruc.2020.129143.

14. CLC Drug Discovery Workbench, 2015, QIAGEN Aarhus A/S, Silkeborgvej 2 Prismet DK-8000 Aarhus C Denmark.

15. Spartan'14; Wavefunction, Inc.: Irvine, CA, USA, 2013.

16. Witvrouw, M.; Daelemans, D.; Pannecouque, C.; Neyts, J.; Andrei, G.; Snoeck, R.; Vandamme, A.-M.; Balzarini, J.; Desmyter, J.; Baba, M.; et al. Broad-Spectrum Antiviral Activity and Mechanism of Antiviral Action of the Fluoroquinolone Derivative K-12. Antivir. Chem. Chemother. 1998, 9, 403-411, doi:10.1177/095632029800900504.

17. Wang, R.; Xu, K.; Shi, W. Quinolone derivatives: Potential anti-HIV agent-development and application. Arch. Pharm. Chem. Life Sci. 2019, 352, 1900045.

18. Fu, L.; Ye, F.; Feng, Y.; Yu, F.; Wang, Q.; Wu, Y.; Zhao, C.; Sun, H.; Huang, B.; Niu, P.; et al. Both Boceprevir and GC376 efficaciously inhibit SARS-CoV-2 by targeting its main protease. Nat. Commun. 2020, 11, 1-8, doi:10.1038/s41467-020-18233-x.

19. Ramírez, D.; Caballero, J. Is It Reliable to Take the Molecular Docking Top Scoring Position as the Best Solution without Considering Available Structural Data? Molecular 2018, 23, 1038, doi:10.3390/molecules23051038. 